\title{
RANDOM INTERSECTION GRAPH PROCESS
}

\author{
Mindaugas Bloznelis ${ }^{1}$ and Michał Karoński \\ ${ }^{I}$ Faculty of Mathematics and Informatics, Vilnius University, Vilnius, Lithuania \\ ${ }^{2}$ Faculty of Mathematics and Computer Science, Adam Mickiewicz University, \\ Poznań, Poland
}

\begin{abstract}
Vertices of an affiliation network are linked to features and two vertices are declared adjacent whenever they share a common feature. We introduce a random intersection graph process aimed at modeling sparse evolving affiliation networks. We establish the asymptotic degree distribution and provide explicit asymptotic formulas for assortativity and clustering coefficients and show how these edge dependence characteristics vary over time.
\end{abstract}

\section{INTRODUCTION}

Affiliation networks are an important class of social networks: members of a network tend to establish relations if they share some common interests or features [12, 17]. For example, for marketing purposes, customers of an internet shop (users of a video-sharing website) can be considered related to each other if they have purchased the same or similar items (e.g., downloaded the same movie). In this article we consider the fitness model of an affiliation network. Vertices (members of the network) and features are assigned independent fitness factors, and a vertex is linked to a feature with probability proportional to the product of their fitness factors. This probability also depends on the arrival times of the vertex and the feature and is set at zero whenever the difference between these arrival times exceeds some threshold value. We show that the network model admits a power-law degree distribution and nontrivial clustering and assortativity coefficients.

Given nonnegative weights $x=\left\{x_{i}\right\}_{i \geq 1}$ and $y=\left\{y_{j}\right\}_{j \geq 1}$, and a nondecreasing positive sequence $\{\tau(t)\}_{t \geq 1}$ satisfying $\lim _{t \rightarrow+\infty} \tau(t)=+\infty$, let $H_{x, y}$ be the random bipartite graph with bipartition $V=\left\{v_{1}, v_{2}, \ldots\right\}$ and $W=\left\{w_{1}, w_{2}, \ldots\right\}$, where edges $\left\{w_{i}, v_{j}\right\}$ are inserted independently and with probabilities

$$
p_{i j}=\min \left\{1, \frac{x_{i} y_{j}}{\sqrt{i j}}\right\} \mathbb{I}_{\{a \tau(j) \leq i \leq b \tau(j)\}} .
$$

Here, $b>a>0$ are fixed numbers. $H_{x, y}$ defines the graph $G_{x, y}$ on the vertex set $V$ such that any $u, v \in V$ are declared adjacent (denoted $u \sim v$ ) whenever they have a common neighbor in $H_{x, y}$.

Address correspondance to Mindaugas Bloznelis, Faculty of Mathematics and Informatics, Vilnius University, Naugarduko 24, Vilnius 03225, Lithuania. E-mail: mindaugas.bloznelis@mif.vu.lt

This article complements and extends the article presented at the 10th Workshop on Algorithms and Models for the Web Graph (WAW 2013) [7]. 
Consider, for example, a library where items $w_{1}, w_{2}, \ldots$ are acquired one after another, and where users $v_{1}, v_{2}, \ldots, v_{j}, \ldots$ are registered at times $j=1,2, \ldots$ User $v_{j}$ picks at random items from the "contemporary literature collection" $[a \tau(j), b \tau(j)]=\left\{w_{i}\right.$ : $a \tau(j) \leq i \leq b \tau(j)\}$ relevant to its arrival time $j$. An item $w_{i}$ is picked with probability proportional to the activity $y_{j}$ of the user $v_{j}$ and the attractiveness $x_{i}$ of the item $w_{i}$, cf. (1.1). The realized bipartite graph $H_{x, y}$ represents "library" records, whereas realized $G_{x, y}$ represents adjacency relations between users in the resulting affiliation network.

Theoretical analysis of such a network becomes simpler if we impose some regularity conditions on the weight sequences $x$ and $y$. A convenient assumption is that $x$ and $y$ are realized values of i.i.d sequences $X=\left\{X_{i}\right\}_{i \geq 1}$ and $Y=\left\{Y_{j}\right\}_{j \geq 1}$. In this way we obtain random graphs $H_{X, Y}$ and $G_{X, Y}$. The parameters of the model are the probability distributions of $X_{1}, Y_{1}$, function $\tau$ (the speed of item acquisition), and cutoffs $a<b$, which, together with $\tau(j)$, determine the interval $[a \tau(j), b \tau(j)]$, which we also interpret as the lifespan of a user $v_{j}$. In what follows, we mainly consider the case where $\tau(t)=t^{v}, v>0$. In this case our choice of weights $1 / \sqrt{i j}$ (together with moment conditions on $X$ and $Y$ ) ensures that the resulting random graph $G_{X, Y}$ is sparse, i.e., the degree sequence $\left\{d\left(v_{j}\right)\right\}_{j \geq 1}$ is stochastically bounded.

Random graph $G_{X, Y}$ is aimed at modeling sparse affiliation networks that evolve in time, such as, e.g., the actor network, where two actors are declared adjacent whenever they have played in the same movie, a collaboration network where two authors are declared adjacent if they have coauthored an article. Such networks display a natural bipartite structure: actors are linked to films, authors are linked to articles. An underlying bipartite structure seems to be present in many social networks: members of a network become acquainted because they share some common interests. The bipartite structure is helpful in explaining the clustering property (network transitivity), and the observed fact that degrees of adjacent vertices are positively correlated (network assortativity), [17, 20].

We note that each vertex of the graph $G_{X, Y}$ can be identified with the random subset of $W$, consisting of items selected by that vertex, and two vertices are adjacent in $G_{X, Y}$ whenever their subsets intersect. Graphs describing such adjacency relations between members of a finite family $V_{n}=\left\{v_{1}, \ldots, v_{n}\right\}$ of random subsets of a given finite set $W_{m}=\left\{w_{1}, \ldots, w_{m}\right\}$ have been introduced in [14], see also [10]. They are called random intersection graphs. We remark that random intersection graphs reproduce empirically observed clustering properties of the actor network with remarkable accuracy ([2], [6]). Unfortunately, they do not account for the evolving nature of the network (actors who acted in 2013 are unlikely to be adjacent to those who acted in 1913) and, therefore, cannot explain how various characteristics of an evolving network vary over time. This drawback of the static model has motivated our interest in evolving random intersection graph $G_{X, Y}$. A related empirical study showing how various characteristics of an evolving collaboration network changed over a 100-year period is presented in [15], see also [16].

The random graph $G_{X, Y}$ can be considered as a random process evolving in time, where the vertex $v_{t}$ that arrived at time $t$ can establish adjacency relations only with contemporaries $v_{s}$ such that the intervals $[a \tau(t), b \tau(t)]$ and $[a \tau(s), b \tau(s)]$ (lifespans of $v_{t}$ and $\left.v_{s}\right)$ intersect. We show that the random intersection graph process admits an asymptotic power-law distribution of the degree $d\left(v_{t}\right)$ of a vertex $v_{t}$ as $t \rightarrow+\infty$. Moreover, we give an explicit description of the asymptotic degree distribution. Furthermore, we show that $G_{X, Y}$ admits a nontrivial clustering and assortativity coefficients and calculate their first-order asymptotics. 
The intuition behind these results is explained as follows. We first observe that in choosing inhomogeneous weight sequences $x$ and $y$, one may expect to obtain an inhomogeneous degree sequence of the graph $G_{x, y}$ : vertices with larger weights attract larger numbers of neighbors. Consequently, in the case where the probability distributions of $X_{1}$ and $Y_{1}$ have heavy tails, we obtain a heavy-tailed asymptotic degree distribution. Second, we observe that if the set $W(t)$ of items selected by a user $v_{t}$ is (stochastically) bounded and the lifespans of two neighbors of $v_{t}$, say $v_{s}$ and $v_{u}$, intersect, then, with a nonvanishing probability, $v_{s}$ and $v_{u}$ share an item from $W(t)$. Consequently, the conditional probability $\alpha_{t \mid s u}=\mathbf{P}\left(v_{s} \sim v_{u} \mid v_{s} \sim v_{t}, v_{t} \sim v_{u}\right)$, called the clustering coefficient, is positive and bounded away from zero. In particular, the underlying bipartite graph structure serves as a clustering mechanism. A similar argument applies to the assortativity coefficient (Pearson's correlation coefficient between degrees of adjacent vertices)

$$
r_{s, t}=\frac{\mathbf{E}_{s t} d\left(v_{s}\right) d\left(v_{t}\right)-\mathbf{E}_{s t} d\left(v_{s}\right) \mathbf{E}_{s t} d_{v}(t)}{\sqrt{\operatorname{Var}_{s t} d\left(v_{s}\right) \operatorname{Var}_{s t} d\left(v_{t}\right)}}
$$

Here, an item shared by adjacent vertices $v_{s} \sim v_{t}$ attracts a number of common neighbors of $v_{s}$ and $v_{t}$. This makes the correlation coefficient positive and bounded away from zero. Here, $\mathbf{E}_{s t}$ denotes the conditional expectation given the event $v_{s} \sim v_{t}$ and $\operatorname{Var}_{s t} d\left(v_{s}\right)=$ $\mathbf{E}_{s t} d^{2}\left(v_{s}\right)-\left(\mathbf{E}_{s t} d_{v}(s)\right)^{2}$.

\section{RESULTS}

\subsection{Degree}

For $\tau(t)$ growing linearly in $t$ we obtain a compound probability distribution in the limit. We remark that in this case $G_{X, Y}$ admits nontrivial asymptotic clustering coefficients, see (2.6), (2.7), (2.8) below. For $\tau(t)$ growing faster than linearly in $t$, we obtain a mixed Poisson asymptotic degree distribution. In this case clustering coefficients vanish.

Theorem 2.1. Let $b>a>0$. Let $\tau(t)=t$. Suppose that $\mathbf{E} X_{1}^{2}<\infty$ and $\mathbf{E} Y_{1}<\infty$. Set $\lambda_{1}=\lambda_{1}\left(Y_{1}\right)=2\left(b^{1 / 2}-a^{1 / 2}\right)\left(\mathbf{E} X_{1}\right) Y_{1}$ and $\lambda_{2}=\lambda_{2}\left(X_{1}\right)=2\left(a^{-1 / 2}-b^{-1 / 2}\right)\left(\mathbf{E} Y_{1}\right) X_{1}$.

For $t \rightarrow+\infty$, the random variable $d\left(v_{t}\right)$ converges in distribution to the random variable

$$
d_{*}=\sum_{j=1}^{\Lambda_{1}} \varkappa_{j},
$$

where $\varkappa_{1}, \varkappa_{2}, \ldots$ are independently and identically distributed random variables independent of the random variable $\Lambda_{1}$. They are distributed as follows. For $r=0,1,2, \ldots$, we have

$\mathbf{P}\left(\varkappa_{1}=r\right)=\frac{r+1}{\mathbf{E} \Lambda_{2}} \mathbf{P}\left(\Lambda_{2}=r+1\right), \quad$ where $\quad \mathbf{P}\left(\Lambda_{i}=r\right)=\mathbf{E} e^{-\lambda_{i}} \frac{\lambda_{i}^{r}}{r !}, \quad i=1,2$.

We remark that the second moment condition $\mathbf{E} X_{1}^{2}<\infty$ of Theorem 2.1 seems to be redundant and could perhaps be waived. 
Theorem 2.2. Let $b>a>0$ and $v>1$. Let $\tau(t)=t^{v}$. Suppose that $\mathbf{E} X_{1}^{2}<\infty$ and $\mathbf{E} Y_{1}<\infty$. Set $\lambda_{3}=\lambda_{3}\left(Y_{1}\right)=\gamma\left(\mathbf{E} X_{1}^{2}\right)\left(\mathbf{E} Y_{1}\right) Y_{1}$, where $\gamma=4 v\left(b^{1 / 2 v}-a^{1 / 2 v}\right)\left(a^{-1 / 2 v}-\right.$ $\left.b^{-1 / 2 v}\right)$.

For $t \rightarrow+\infty$, the random variable $d\left(v_{t}\right)$ converges in distribution to the random variable $\Lambda_{3}$ having the probability distribution

$$
\mathbf{P}\left(\Lambda_{3}=r\right)=\mathbf{E} e^{-\lambda_{3}} \frac{\lambda_{3}^{r}}{r !}, \quad r=0,1,2, \ldots
$$

Remark 2.3. The probability distributions $\Lambda_{i}, i=1,2,3$, are Poisson mixtures. One way to sample from the distribution of $\Lambda_{i}$ is to generate random variable $\lambda_{i}$ and then, given $\lambda_{i}$, to generate a Poisson random variable with the parameter $\lambda_{i}$. In Theorem 2.1 we obtain a power-law asymptotic degree distribution provided that the heavier of the tails $t \rightarrow \mathbf{P}\left(\varkappa_{1}>t\right)$ and $t \rightarrow \mathbf{P}\left(\Lambda_{1}>t\right)$ has a power law, see [9]. In Theorem 2.2 we obtain a power-law asymptotic degree distribution provided that $Y_{1}$ has a power law. Indeed, since for large $\lambda$ the Poisson distribution $\mathcal{P}(\lambda)$ is tightly concentrated around its mean $\lambda$, we have that, given a large value of $Y_{1}$, the conditional distribution of $\Lambda_{3}$ is concentrated around $\lambda_{3}=\gamma\left(\mathbf{E} X_{1}^{2}\right)\left(\mathbf{E} Y_{1}\right) Y_{1}$. Now, since the latter random variable has a power law, we conclude that $\Lambda_{3}$ has a power law with the same exponent as $Y_{1}$.

Remark 2.4. The result of Theorem 2.2 extends to a more general class of increasing nonnegative functions $\tau$. In particular, assuming that

$$
\lim _{t \rightarrow+\infty} \frac{t}{\tau(t)}=0, \quad \sup _{t>1} \frac{\tau^{-1}(2 t)}{\tau^{-1}(t)}<\infty,
$$

and that there exists a finite limit

$$
\gamma^{*}=\lim _{t \rightarrow+\infty} t^{-1 / 2} \sum_{a \tau(t) \leq i \leq b \tau(t)} i^{-1} \sum_{j: a \tau(j) \leq i \leq b \tau(j)} j^{-1 / 2},
$$

we obtain the convergence in distribution of $d\left(v_{t}\right)$ to $\Lambda_{3}$ defined by (2.3) with $\lambda_{3}=$ $\gamma^{*}\left(\mathbf{E} X_{1}^{2}\right)\left(\mathbf{E} Y_{1}\right) Y_{1}$. Here, $\tau^{-1}$ denotes the inverse of $\tau$ (i.e., $\tau\left(\tau^{-1}(t)\right)=t$ ).

Remark 2.5. The function $\tau(t)=t \ln t$, which grows slower than any power $t^{v}, v>1$, satisfies conditions of Remark 2.4 with $\gamma^{*}=4\left(a^{-1 / 2}-b^{-1 / 2}\right)\left(b^{1 / 2}-a^{1 / 2}\right)$. Furthermore, the functions $\tau_{1}(t)=e^{\ln ^{2} t}$ and $\tau_{2}(t)=e^{t}$, which grow faster than any power $t^{\nu}$, satisfy conditions of Remark 2.4 with $\gamma^{*}=0$ and now the asymptotic degree distribution is degenerate. The function $\tau(t)=t^{\nu}$, for $0<v<1$, does not satisfy conditions of Remark 2.4. In this case, the asymptotic degree distribution is again degenerate, i.e., for $\mathbf{E} X_{1}<\infty$ and $\mathbf{E} Y_{1}<\infty$ we have $d\left(v_{t}\right)=o_{P}(1)$ as $t \rightarrow+\infty$. This fact is obtained in the same way as the statement (i) of Theorem 1 of [3].

\subsection{Clustering}

We assume that $s<t<u$ and consider the conditional probabilities $\alpha_{s \mid t u}, \alpha_{t \mid s u}$ and $\alpha_{u \mid s t}$. These probabilities are all different and, given $0<a<b$, they depend mainly on the ratios $s / t, s / u$, and $t / u$. Denote $p_{\Delta}=p_{\Delta}(s, t, u)=\mathbf{P}\left(v_{s} \sim v_{t}, v_{s} \sim v_{u}, v_{t} \sim v_{u}\right)$ the probability that $v_{s}, v_{t}, v_{u}$ make up a triangle. 
Theorem 2.6. Let $b>a>0$. Let $\tau(t)=t$. Suppose that $\mathbf{E} X_{1}^{3}<\infty$ and $\mathbf{E} Y_{1}^{2}<\infty$. Assume that $s, t, u \rightarrow+\infty$ so that $s<t<u$ and $\lceil a u\rceil \leq\lfloor b s\rfloor$. We have

$$
\begin{aligned}
p_{\Delta} & =\frac{a_{3} b_{1}^{3}}{\sqrt{s t u}}\left(\frac{2}{\sqrt{a u}}-\frac{2}{\sqrt{b s}}\right)+o\left(t^{-2}\right), \\
\alpha_{t \mid s u} & =\frac{p_{\Delta}}{p_{\Delta}+a_{2}^{2} b_{1}^{2} b_{2} t^{-1}(s u)^{-1 / 2} \delta_{t \mid s u}}+o(1), \\
\alpha_{s \mid t u} & =\frac{p_{\Delta}}{p_{\Delta}+a_{2}^{2} b_{1}^{2} b_{2} s^{-1}(t u)^{-1 / 2} \delta_{s \mid t u}}+o(1), \\
\alpha_{u \mid s t} & =\frac{p_{\Delta}}{p_{\Delta}+a_{2}^{2} b_{1}^{2} b_{2} u^{-1}(s t)^{-1 / 2} \delta_{u \mid s t}}+o(1) .
\end{aligned}
$$

Here, we denote $a_{i}=\mathbf{E} X_{1}^{i}, i=2,3$, and $b_{j}=\mathbf{E} Y_{1}^{j}, j=1,2$, and

$$
\begin{aligned}
& \delta_{t \mid s u}=\ln (u / t) \ln (t / s)+\ln (u / t) \ln (b s / a u)+\ln (t / s) \ln (b s / a u)+\ln ^{2}(b s / a u), \\
& \delta_{s \mid t u}=\ln (u / t) \ln (b s / a u)+\ln ^{2}(b s / a u), \\
& \delta_{u \mid s t}=\ln (t / s) \ln (b s / a u)+\ln ^{2}(b s / a u) .
\end{aligned}
$$

We remark that the condition $\lceil a u\rceil \leq\lfloor b s\rfloor$ of Theorem 2.6 excludes the trivial case where $p_{\Delta} \equiv 0$. Indeed, for $s<u$, the converse inequality $\lceil a u\rceil>\lfloor b s\rfloor$ means that the lifetimes of $v_{s}$ and $v_{u}$ do not intersect and, therefore, we have $\mathbf{P}\left(v_{s} \sim v_{u}\right) \equiv 0$. In addition, the inequality $\lceil a u\rceil \leq\lfloor b s\rfloor$ implies that positive numbers $\delta_{t \mid s u}, \delta_{s \mid t u}, \delta_{u \mid s t}$ are bounded from above by a constant (depending only on $a$ and $b$ ).

\subsection{Assortativity}

We assume that $\tau(t)=t$ and consider the sequence of random variables $\left\{d\left(v_{t}\right)\right\}_{t \geq 1}$. From Theorem 2.1 we know about the possible limiting distributions for $d\left(v_{t}\right)$. Moreover, from the fact that $G_{X, Y}$ is sparse, we can conclude that, for any given $k$, the random variables $d\left(v_{t}\right), d\left(v_{t+1}\right), \ldots, d\left(v_{t+k}\right)$ are asymptotically independent as $t \rightarrow+\infty$. An interesting question is about the statistical dependence between $d\left(v_{s}\right)$ and $d\left(v_{t}\right)$ if we know, in addition, that vertices $v_{s}$ and $v_{t}$ are adjacent in $G_{X, Y}$. We assume that $s<t$ and let $s, t \rightarrow+\infty$ so that $b s-a t \rightarrow+\infty$. Note that the latter condition ensures that the shared lifetime of $v_{s}$ and $v_{t}$ tends to infinity as $s, t \rightarrow+\infty$. In this case we obtain that conditional moments

$$
\begin{aligned}
& \mathbf{E}_{s t} d\left(v_{s}\right)=\mathbf{E}_{s t} d\left(v_{t}\right)+o(1)=\delta_{1}+o(1), \\
& \mathbf{E}_{s t} d^{2}\left(v_{s}\right)=\mathbf{E}_{s t} d^{2}\left(v_{t}\right)+o(1)=\delta_{2}+o(1), \\
& \mathbf{E}_{s t} d\left(v_{s}\right) d\left(v_{t}\right)=\delta_{2}-\Delta+o(1)
\end{aligned}
$$

are asymptotically constant. Here, $\Delta=h_{1}^{-1}\left(2 h_{3}+2 h_{5}+4\left(h_{6}-h_{7}\right)\right)$ and

$$
\delta_{1}=1+h_{1}^{-1}\left(h_{2}+2 h_{3}\right), \quad \delta_{2}=1+h_{1}^{-1}\left(3 h_{2}+6 h_{3}+h_{4}+6 h_{5}+4 h_{6}\right) .
$$


Furthermore, the quantities $h_{i}$ are polynomials in moments $a_{k}=\mathbf{E} X_{1}^{k}$ and $b_{k}=\mathbf{E} Y_{1}^{k}$

$$
\begin{aligned}
& h_{1}=a_{2} b_{1}^{2}, \quad h_{2}=a_{3} b_{1}^{3} \tilde{\gamma}, \quad h_{3}=a_{2}^{2} b_{1}^{2} b_{2} \tilde{\gamma}(\sqrt{b}-\sqrt{a}), \\
& h_{4}=a_{4} b_{1}^{4} \tilde{\gamma}^{2}, \quad h_{5}=a_{2} a_{3} b_{1}^{3} b_{2} \tilde{\gamma}^{2}(\sqrt{b}-\sqrt{a}), \\
& h_{6}=a_{2}^{3} b_{1}^{3} b_{3} \tilde{\gamma}^{2}(\sqrt{b}-\sqrt{a})^{2}, \quad h_{7}=a_{2}^{3} b_{1}^{2} b_{2}^{2} \tilde{\gamma}^{2}(\sqrt{b}-\sqrt{a})^{2},
\end{aligned}
$$

and $\tilde{\gamma}=2\left(a^{-1 / 2}-b^{-1 / 2}\right)$.

From (1.2) and (2.9) we obtain that the assortativity coefficient

$$
r_{s t}=1-\frac{\Delta}{\delta_{2}-\delta_{1}^{2}}+o(1)
$$

is asymptotically constant.

\subsection{Related Work}

The degree distribution of the typical vertex of finite random intersection graphs has been studied by several authors, see, e.g., [2], [8], [13], [18], the clustering properties have been studied in [2], [5] [8], [11]. Assortativity coefficient has been evaluated in [5].

In a recent article [4], a related intersection graph process is considered, where a newly arrived user is linked preferentially to already highly popular items, thus, further increasing their popularity/attractiveness measured by the number of users that have chosen this item. We note that the spatial preferred attachment model [1] can also be viewed as a geometric intersection graph process.

\section{PROOFS}

We first introduce some notation. Then we prove Theorems 2.1 and 2.2. The proof of Remark 2.4 goes along the lines of the proof of Theorem 2.2 and is omitted. Theorem 2.6 and relations (2.9) are shown in the accompanying study [7] (see pp. 100-104 of [7]). In order to avoid repetition we do not present their proofs here.

In the proofs of Theorems 2.1 and 2.2, we apply the approach used in [3]. Here, the main technical difference between the static model $G\left(P_{1}, P_{2}, n, m\right)$ of [3] and the process $G_{X, Y}$ is that the scaling $\frac{1}{\sqrt{i j}}$ of the edge inclusion probability $p_{i j}$ of the underlying bipartite graph of $G_{X, Y}$ depends on the arrival times $i$ and $j$ of item $w_{i}$ and user $v_{j}$, whereas the scaling $\frac{1}{\sqrt{n m}}$ of the corresponding edge inclusion probability $p_{i j}^{\prime}$ of the static model is independent of $i$ and $j$. The dependence on arrival times $i$ and $j$ adds some more complexity to the proofs and affects the parameters of random variables $\lambda_{1}, \lambda_{2}, \lambda_{3}$ of (2.2), (2.3).

We denote $a_{k}=\mathbf{E} X_{1}^{k}$ and $b_{k}=\mathbf{E} Y_{1}^{k}$. Throughout the proof, limits are taken as $t \rightarrow+\infty$, if not stated otherwise. By $c$ we denote positive numbers that may depend only on $a, b$ and $\tau$. We remark that $c$ may attain different values in different places. We say that a sequence of random variables $\left\{\zeta_{t}\right\}_{t \geq 1}$ converges to zero in probability (denoted $\left.\zeta_{t}=o_{P}(1)\right)$ whenever $\lim \sup _{t} \mathbf{P}\left(\left|\zeta_{t}\right|>\varepsilon\right)=0$ for each $\varepsilon>0$. The sequence $\left\{\zeta_{t}\right\}_{t \geq 1}$ is called stochastically bounded (denoted $\zeta_{t}=O_{P}(1)$ ) whenever for each $\delta>0$ there exists $N_{\delta}>0$ such that $\limsup _{t} \mathbf{P}\left(\left|\zeta_{t}\right|>N_{\delta}\right)<\delta$.

Time intervals

$$
T_{t}=\{i: a \tau(t) \leq i \leq b \tau(t)\}, \quad T_{i}^{*}=\{j: a \tau(j) \leq i \leq b \tau(j)\}
$$


can be interpreted as lifetimes of the actor $v_{t}$ and attribute $w_{i}$ respectively. Here and below elements of $V$ are called actors, elements of $W$ are called attributes. The oldest and youngest actors that may establish a communication link with $v_{t}$ are denoted $v_{t_{-}}$and $v_{t_{+}}$. Here

$$
t_{-}=\min \left\{j: T_{j} \cap T_{t} \neq \emptyset\right\}, \quad t_{+}=\max \left\{j: T_{j} \cap T_{t} \neq \emptyset\right\} .
$$

The event "edge $\left\{w_{i}, v_{j}\right\}$ is present in $H_{X, Y}$ " is denoted $w_{i} \rightarrow v_{j}$. Introduce random variables

$$
\begin{aligned}
\mathbb{I}_{i j} & =\mathbb{I}_{\left\{w_{i} \rightarrow v_{j}\right\}}, \quad \mathbb{I}_{i}=\mathbb{I}_{i t}, \quad U_{i}=\sum_{j \in T_{i}^{*} \backslash\{t\}} \mathbb{I}_{i j}, \quad L=L_{t}=\sum_{i \in T_{t}} U_{i} \mathbb{I}_{i}, \\
B_{k}(I) & =\sum_{j \in I} Y_{j}^{k} j^{-k / 2}, \quad A_{k}(I)=\sum_{i \in I} X_{i}^{k} i^{-k / 2}, \quad I \subset \mathbb{N}, \\
\lambda_{i j} & =X_{i} Y_{j} / \sqrt{i j}, \quad Q_{X Y}(t)=\sum_{i \in T_{t}} \lambda_{i t} \sum_{j \in T_{i}^{*} \backslash\{t\}} \lambda_{i j} \min \left\{1, \lambda_{i j}\right\} .
\end{aligned}
$$

We remark that $U_{i}$ counts all neighbors of $w_{i}$ in $H_{X, Y}$ belonging to the set $V \backslash\left\{v_{t}\right\}$, and $L_{t}$ counts all paths of length 2 in $H_{X, Y}$ starting from $v_{t}$. Introduce events

$$
\mathcal{A}_{t}=\left\{\lambda_{i t} \leq 1, i \in T_{t}\right\}, \quad \mathcal{B}_{t}(\varepsilon)=\left\{Y_{j} \leq \varepsilon^{2} j, j \in\left[t_{-}, t_{+}\right] \backslash\{t\}\right\}, \quad \varepsilon>0 .
$$

By $\tilde{\mathbf{P}}$ and $\tilde{\mathbf{E}}$ we denote the conditional probability and expectation given $X, Y$. The conditional probability and expectation given $Y$ is denoted $\mathbf{P}_{X}$ and $\mathbf{E}_{X}$. By $\mathbf{P}_{t}$ and $\mathbf{E}_{t}$ we denote the conditional probability and expectation given $Y_{t}$. By $d_{T V}(\zeta, \xi)$ we denote the total variation distance between the probability distributions of random variables $\zeta$ and $\xi$. In the case where $\zeta, \xi$ and $X, Y$ are defined on the same probability space, we denote by $\tilde{d}_{T V}(\zeta, \xi)$ the total variation distance between the conditional distributions of $\zeta$ and $\xi$ given $X, Y$.

In the proof, we use the following simple fact. For a uniformly bounded sequence of nonnegative random variables $\left\{\zeta_{t}\right\}_{t \geq 1}$ (i.e., such that for some nonrandom $h>0$ and each $t$ the inequalities $0 \leq \zeta_{t}<h$ hold almost surely) we have

$$
\zeta_{t}=o_{P}(1) \quad \Rightarrow \quad \mathbf{E} \zeta_{t}=o(1)
$$

In particular, given a sequence of bivariate random vectors $\left\{\left(\phi_{t}, \psi_{t}\right)\right\}_{t \geq 1}$, defined on the same probability space as $X, Y$, we have

$$
\tilde{d}_{T V}\left(\phi_{t}, \psi_{t}\right)=o_{P}(1) \quad \Rightarrow \quad d_{T V}\left(\phi_{t}, \psi_{t}\right)=o(1) .
$$

Finally, we note that the notation introduced in the proof of a particular lemma or theorem is valid only for that proof.

\subsection{Proof of Theorem 2.1}

Before the proof we collect auxiliary results. For $\tau(t):=t$ and $T_{t}, T_{i}^{*}$ defined in (3.1), we have

$$
\begin{array}{rlrl}
\sum_{i \in T_{t}} i^{-1 / 2} & =t^{1 / 2} \gamma_{1}+r t^{-1 / 2}, & \sum_{j \in T_{i}^{*}} j^{-1 / 2} & =i^{1 / 2} \gamma_{2}+r^{\prime} i^{-1 / 2}, \\
\gamma_{1}:=2\left(b^{1 / 2}-a^{1 / 2}\right), & \gamma_{2}:=2\left(a^{-1 / 2}-b^{-1 / 2}\right),
\end{array}
$$


where $|r|,\left|r^{\prime}\right| \leq c$.

Lemma 3.1. Let $t \rightarrow+\infty$. Assume that $\mathbf{E} X_{1}^{2}<\infty$ and $\mathbf{E} Y_{1}<\infty$. We have

$$
\begin{gathered}
\forall \varepsilon>0 \quad \mathbf{P}\left(\mathcal{B}_{t}(\varepsilon)\right)=1-o(1), \\
t^{-1} B_{2}\left(\left[t_{-}, t_{+}\right] \backslash\{t\}\right)=o_{P}(1), \\
\mathbf{P}\left(d\left(v_{t}\right) \neq L_{t}\right)=o(1), \\
\mathbf{P}\left(\mathcal{A}_{t}\right)=1-o(1), \\
Q_{X Y}(t)=o_{P}(1), \quad \mathbf{E} Q_{X Y}(t)=o(1) .
\end{gathered}
$$

For any integers $t>a^{-1}\left(b+b^{-1}\right)$ and $i \in T_{t}$, and any $0<\varepsilon<1$ we have

$$
\begin{aligned}
& \left|\mathbf{E} A_{1}\left(T_{t}\right)-a_{1} \gamma_{1} t^{1 / 2}\right| \leq c a_{1} t^{-1 / 2}, \quad\left|\mathbf{E} B_{1}\left(T_{i}^{*} \backslash\{t\}\right)-b_{1} \gamma_{2} i^{1 / 2}\right| \leq c b_{1} i^{-1 / 2}, \\
& \mathbf{E}\left|B_{1}\left(T_{i}^{*} \backslash\{t\}\right)-b_{1} \gamma_{2} i^{1 / 2}\right| \mathbb{I}_{\mathcal{B}_{t}(\varepsilon)} \leq c i^{1 / 2}\left(\varepsilon b_{1}^{1 / 2}+\mathbf{E} Y_{1} \mathbb{I}_{\left\{Y_{1}>\varepsilon^{2} t_{-}\right\}}\right)+c b_{1} i^{-1 / 2}, \\
& \mathbf{E}\left|A_{1}\left(T_{t}\right)-a_{1} \gamma_{1} t^{1 / 2}\right| \leq c a_{2}^{1 / 2} .
\end{aligned}
$$

Proof of Lemma 3.1. Proof of (3.7). We estimate the probability of the complement event $\overline{\mathcal{B}}_{t}(\varepsilon)$ using the union bound and Markov's inequality

$$
\mathbf{P}\left(\overline{\mathcal{B}}_{t}(\varepsilon)\right) \leq \sum_{t_{-} \leq j \leq t_{+}} \mathbf{P}\left(Y_{j}>\varepsilon^{2} j\right)=t_{+} \mathbf{P}\left(Y_{1}>\varepsilon^{2} t_{-}\right) \leq \varepsilon^{-2}\left(t_{+} / t_{-}\right) \mathbf{E} Y_{1} \mathbb{I}_{\left\{Y_{1}>\varepsilon^{2} t_{-}\right\}}=o(1) .
$$

Here we estimate $t_{+} / t_{-} \leq c$ and invoke the bound $\mathbf{E} Y_{1} \mathbb{I}_{\left\{Y_{1}>s\right\}}=o(1)$, for $s \rightarrow+\infty$.

Proof of (3.8). Denote $\hat{b}_{2}(t)=t^{-2} \sum_{1 \leq j \leq t} Y_{j}^{2}$. We note that $\mathbf{E} Y_{1}<\infty$ implies $\hat{b}_{2}(t)=o_{P}(1)$. The latter bound in combination with the simple inequality $t_{+} / t_{-} \leq c$ implies (3.8).

Proof of (3.10). Let $\overline{\mathcal{A}}_{t}$ denote the complement event to $\mathcal{A}_{t}$. We have, by the union bound and Markov's inequality,

$$
\mathbf{P}_{t}\left(\overline{\mathcal{A}}_{t}\right) \leq \sum_{i \in T_{t}} \mathbf{P}_{t}\left(\lambda_{i t} \geq 1\right) \leq \sum_{i \in T_{t}}(i t)^{-1} Y_{t}^{2} a_{2} \leq c a_{2} t^{-1} Y_{t}^{2}
$$

We obtain the bound $\mathbf{P}_{t}\left(\overline{\mathcal{A}}_{t}\right)=o(1)$, which implies (3.10), see (3.4).

Proof of (3.9). In view of (3.4) it suffices to show that $\mathbf{P}_{X}\left(d\left(v_{t}\right) \neq L_{t}\right)=o_{P}(1)$. We note that $d\left(v_{t}\right) \neq L_{t}$ if and only if $S \geq 1$, where $S=\sum^{\prime} \mathbb{I}_{i_{1}} \mathbb{I}_{i_{2}} \mathbb{I}_{i_{1} j} \mathbb{I}_{i_{2} j}$. Here, we denote $\sum^{\prime}=\sum_{\left\{i_{1}, i_{2}\right\} \subset T_{t}} \sum_{j \in T_{i_{1}}^{*} \cap T_{i_{2}}^{*}, j \neq t}$. Observing that

$$
\mathbf{E}_{X} \mathbb{I}_{i_{1}} \mathbb{I}_{i_{2}} \mathbb{I}_{i_{1} j} \mathbb{I}_{i_{2} j}=\mathbf{E}_{X} p_{i_{1} t} p_{i_{2} t} p_{i_{1} j} p_{i_{2} j} \leq a_{2}^{2} Y_{t}^{2} Y_{j}^{2} /\left(i_{1} i_{2} t j\right),
$$

we obtain, by Markov's inequality,

$$
\mathbf{P}_{X}\left(d\left(v_{t}\right) \neq L_{t}\right)=\mathbf{P}_{X}(S \geq 1) \leq \mathbf{E}_{X} S \leq a_{2}^{2} Y_{t}^{2} t^{-1} \sum^{\prime} Y_{j}^{2}\left(i_{1} i_{2} j\right)^{-1} .
$$

The simple bound $\sum_{\left\{i_{1}, i_{2}\right\} \subset T_{t}} \frac{1}{i_{1} i_{2}} \leq c$ implies $\sum^{\prime} Y_{j}^{2}\left(i_{1} i_{2} j\right)^{-1} \leq c B_{2}\left(\left[t_{-}, t_{+}\right] \backslash\{t\}\right)$. Now, by (3.8) the right-hand side of (3.15) tends to zero in probability. 
Proof of (3.11). Denote $\hat{X}_{i}=\max \left\{X_{i}, 1\right\}, \hat{Y}_{j}=\max \left\{Y_{j}, 1\right\}$, and let $\hat{Q}_{X Y}(t)$ denote the sum in (3.3), where $\lambda_{i j}$ is replaced by $\hat{\lambda}_{i j}=\hat{X}_{i} \hat{Y}_{j} / \sqrt{i j}$. We observe that $\mathbf{E} X_{1}^{2}<\infty$ and $\mathbf{E} Y_{1}<\infty$ imply

$$
a_{\varphi}:=\mathbf{E} \hat{X}_{1}^{2} \varphi\left(\hat{X}_{1}\right)<\infty, \quad b_{\varphi}:=\mathbf{E} \hat{Y}_{1} \varphi\left(\hat{Y}_{1}\right)<\infty,
$$

for some positive increasing function $\varphi:[1,+\infty) \rightarrow[0,+\infty)$ satisfying $\varphi(u) \rightarrow+\infty$ as $u \rightarrow+\infty$ (clearly, $\varphi(\cdot)$ depends on the distributions of $X_{1}$ and $Y_{1}$ ). In addition, we can choose $\varphi$ satisfying $\varphi(u) \leq u$ and $\varphi(s u) \leq \varphi(s) \varphi(u)$, for $s, u \geq 1$. From these inequalities, one derives the inequality $\min \left\{1, \hat{\lambda}_{i j}\right\} \leq \varphi\left(\hat{X}_{i}\right) \varphi\left(\hat{Y}_{j}\right) / \varphi(\sqrt{i j})$ (see formula (13) in [3]). The latter inequality implies

$$
\hat{Q}_{X Y}(t) \leq \hat{Y}_{t} Q_{X Y}^{*}(t), \quad Q_{X Y}^{*}(t):=\sum_{i \in T_{t}} \frac{\hat{X}_{i}^{2} \varphi\left(\hat{X}_{i}\right)}{\sqrt{t i}} \sum_{j \in T_{i}^{*} \backslash\{t\}} \frac{\hat{Y}_{j} \varphi\left(\hat{Y}_{j}\right)}{\sqrt{i j}} \frac{1}{\varphi(\sqrt{i j})} .
$$

Furthermore, for $i \in T_{t}$ and $j \in T_{i}^{*}$ we have $i j \geq\lfloor a t\rfloor t_{-}=: t_{*}^{2}$, and $t_{*} \rightarrow+\infty$ as $t \rightarrow+\infty$. Hence,

$$
\mathbf{E} Q_{X Y}^{*}(t) \leq \frac{1}{\varphi\left(t_{*}\right)} a_{\varphi} b_{\varphi} \sum_{i \in T_{t}} \frac{1}{\sqrt{t i}} \sum_{j \in T_{i}^{*}} \frac{1}{\sqrt{i j}}=O\left(\frac{1}{\varphi\left(t_{*}\right)}\right)=o(1)
$$

This bound together with the inequalities $Q_{X Y}(t) \leq \hat{Q}_{X Y}(t) \leq \hat{Y}_{t} Q_{X Y}^{*}(t)$ shows (3.11).

Proof of (3.12). These inequalities follow from (3.6).

Proof of (3.13). Denote $\Delta=\left|\tilde{b}-b_{1} \gamma_{2} i^{1 / 2}\right|$, where $\tilde{b}$ denotes the sum $B_{1}\left(T_{i}^{*} \backslash\{t\}\right)$, but with $Y_{j}$ replaced by $\tilde{Y}_{j}=Y_{j} \mathbb{I}_{\left\{Y_{j} \leq \varepsilon^{2} j\right\}}, j \in T_{i}^{*} \backslash\{t\}$. We have

$$
\left|B_{1}\left(T_{i}^{*} \backslash\{t\}\right)-b_{1} \gamma_{2} i^{1 / 2}\right| \mathbb{I}_{\mathcal{B}_{t}(\varepsilon)}=\Delta \mathbb{I}_{\mathcal{B}_{t}(\varepsilon)} \leq \Delta \leq \Delta_{1}+\Delta_{2}+\Delta_{3}
$$

where we denote $\Delta_{1}=|\tilde{b}-\mathbf{E} \tilde{b}|, \Delta_{2}=\left|\mathbf{E} \tilde{b}-\mathbf{E} B_{1}\left(T_{i}^{*} \backslash\{t\}\right)\right|, \Delta_{3}=\left|\mathbf{E} B_{1}\left(T_{i}^{*} \backslash\{t\}\right)-b_{1} \gamma_{2} i^{1 / 2}\right|$. Next, we evaluate $\mathbf{E} \Delta_{1}$ and $\Delta_{2}$ :

$$
\begin{aligned}
& \left(\mathbf{E} \Delta_{1}\right)^{2} \leq \mathbf{E}\left(\Delta_{1}^{2}\right) \leq \sum_{j \in T_{i}^{*} \backslash\{t\}} j^{-1} \mathbf{E} \tilde{Y}_{j}^{2} \leq \varepsilon^{2} b_{1}\left|T_{i}^{*}\right| \\
& \Delta_{2} \leq \sum_{j \in T_{i}^{*} \backslash\{t\}} j^{-1 / 2} \mathbf{E} Y_{j} \mathbb{I}_{\left\{Y_{j}>\varepsilon^{2} j\right\}} \leq \mathbf{E} Y_{1} \mathbb{I}_{\left\{Y_{1}>\varepsilon^{2} t_{-}\right\}} \sum_{j \in T_{i}^{*} \backslash\{t\}} j^{-1 / 2} .
\end{aligned}
$$

In (3.17) we first apply Cauchy-Schwartz, then use the linearity of variance of a sum of independent random variables, and finally apply the inequality $\operatorname{Var} \tilde{Y}_{j} \leq \mathbf{E} \tilde{Y}_{j}^{2} \leq j \varepsilon^{2} \mathbf{E} Y_{j}$. Invoking (3.12), (3.17), (3.18) in (3.16) and using (3.6) and $\left|T_{i}^{*}\right| \leq c i$, we obtain (3.13).

Proof of (3.14). We have $\mathbf{E}\left|A_{1}\left(T_{t}\right)-a_{1} \gamma_{1} t^{1 / 2}\right| \leq \mathbf{E} \tilde{\Delta}_{1}+\tilde{\Delta}_{2}$, where $\tilde{\Delta}_{1}:=\mid A_{1}\left(T_{t}\right)-$ $\mathbf{E} A_{1}\left(T_{t}\right) \mid$ and $\tilde{\Delta}_{2}=\left|\mathbf{E} A_{1}\left(T_{t}\right)-a_{1} \gamma_{1} t^{1 / 2}\right|$, and invoke the inequalities

$$
\left(\mathbf{E} \tilde{\Delta}_{1}\right)^{2} \leq \mathbf{E} \tilde{\Delta}_{1}^{2}=\sum_{i \in T_{t}} j^{-1}\left(a_{2}-a_{1}^{2}\right) \leq c a_{2}
$$

and $\tilde{\Delta}_{2} \leq c a_{1} \leq c a_{2}^{1 / 2}$, see (3.12).

Inequality (3.19) below is referred to as Le Cam's inequality, see e.g., [19]. 
Lemma 3.2. Let $S=\mathbb{I}_{1}+\mathbb{I}_{2}+\cdots+\mathbb{I}_{n}$ be the sum of independent random indicators with probabilities $\mathbf{P}\left(\mathbb{I}_{i}=1\right)=p_{i}$. Let $\Lambda$ be a Poisson random variable with mean $p_{1}+\cdots+p_{n}$. The total variation distance between the distributions $P_{S}$ of $S$ and $P_{\Lambda}$ of $\Lambda$

$$
d_{T V}(S, \Lambda):=\sup _{A \subset\{0,1,2 \ldots\}}|\mathbf{P}(S \in A)-\mathbf{P}(\Lambda \in A)| \leq \sum_{i} p_{i}^{2}
$$

Proof of Theorem 2.1. Before the proof we introduce some notation. Given $X, Y$, we generate independent Poisson random variables

$$
\eta_{i}, \quad \xi_{1 i}, \quad \xi_{3 i}, \quad \xi_{4 i}, \quad \Delta_{r i}, \quad i \in T_{t}, \quad r=1,2,3,
$$

with conditional mean values

$$
\begin{aligned}
& \tilde{\mathbf{E}} \eta_{i}=\lambda_{i t}, \quad \tilde{\mathbf{E}} \xi_{1 i}=\sum_{j \in T_{i}^{*} \backslash\{t\}} p_{i j}, \quad \tilde{\mathbf{E}} \xi_{3 i}=X_{i} b_{1} \gamma_{2}, \quad \tilde{\mathbf{E}} \xi_{4 i}=X_{i} \bar{b} i^{-1 / 2}, \\
& \tilde{\mathbf{E}} \Delta_{1 i}=\sum_{j \in T_{i}^{*} \backslash\{t\}}\left(\lambda_{i j}-p_{i j}\right), \quad \tilde{\mathbf{E}} \Delta_{2 i}=X_{i} \delta_{2 i} i^{-1 / 2}, \quad \tilde{\mathbf{E}} \Delta_{3 i}=X_{i} \delta_{3 i} i^{-1 / 2} .
\end{aligned}
$$

Here,

$$
\delta_{2 i}=B_{1}\left(T_{i}^{*} \backslash\{t\}\right)-\bar{b}, \quad \delta_{3 i}=b_{1} \gamma_{2} i^{1 / 2}-\bar{b}, \quad \bar{b}=\min \left\{B_{1}\left(T_{i}^{*} \backslash\{t\}\right), b_{1} \gamma_{2} i^{1 / 2}\right\} .
$$

Finally, we define $\xi_{2 i}=\xi_{1 i}+\Delta_{1 i}, i \in T_{t}$ and introduce random variables

$$
L_{0 t}=\sum_{i \in T_{t}} \eta_{i} U_{i}, \quad L_{r t}=\sum_{i \in T_{t}} \eta_{i} \xi_{r i}, \quad r=1,2,3 .
$$

We assume, in addition, that given $X, Y$, the families of random variables $\left\{\mathbb{I}_{i}, i \in T_{t}\right\}$ and $\left\{\xi_{r i}, i \in T_{t}, r=1,2,3,4\right\}$ are conditionally independent, and that $\left\{\eta_{i}, i \in T_{t}\right\}$ is conditionally independent of the set of edges of $H_{X, Y}$ that are not incident to $v_{t}$.

We are ready to start the proof. In view of (3.9) the random variables $d\left(v_{t}\right)$ and $L_{t}$ have the same asymptotic distribution (if any). We shall prove that $L_{t}$ converges in distribution to $d_{*}$. In the proof we approximate $L_{t}$ by the random variable $L_{3 t}$ (first step of the proof). Afterward we show that $L_{3 t}$ converges in distribution to $d_{*}$ (second step of the proof).

Step 1. In order to show that $L_{t}$ and $L_{3 t}$ have the same asymptotic distribution (if any) we prove the bounds

$$
\begin{aligned}
d_{T V}\left(L_{t}, L_{0 t}\right) & =o(1), & d_{T V}\left(L_{0 t}, L_{1 t}\right)=o(1), \\
\mathbf{E}\left|L_{1 t}-L_{2 t}\right| & =o(1), & \tilde{L}_{2 t}-\tilde{L}_{3 t}=o_{P}(1) .
\end{aligned}
$$

Here $\tilde{L}_{2 t}$ and $\tilde{L}_{3 t}$ are marginals of the random vector $\left(\tilde{L}_{2 t}, \tilde{L}_{3 t}\right)$ constructed in (3.26) following which has the property that $\tilde{L}_{2 t}$ has the same distribution as $L_{2 t}$ and $\tilde{L}_{3 t}$ has the same distribution as $L_{3 t}$.

Let us prove the first bound of (3.21). We shall show that

$$
\tilde{d}_{T V}\left(L_{t}, L_{0 t}\right) \mathbb{I}_{\mathcal{A}_{t}} \leq t^{-1} Y_{t}^{2} A_{2}\left(T_{t}\right)
$$


From the inequality $\mathbf{E} A_{2}\left(T_{t}\right)=\sum_{i \in T_{t}} i^{-1} a_{2} \leq c a_{2}$ we conclude that $A_{2}\left(T_{t}\right)$ is stochastically bounded. Hence $t^{-1} Y_{t}^{2} A_{2}\left(T_{t}\right)=o_{P}(1)$. This bound and (3.23) combined with (3.10) imply

$$
\tilde{d}_{T V}\left(L_{t}, L_{0 t}\right) \leq \tilde{d}_{T V}\left(L_{t}, L_{0 t}\right) \mathbb{I}_{\mathcal{A}_{t}}+\mathbb{I}_{\overline{\mathcal{A}}_{t}}=o_{P}(1) .
$$

Now the first bound of (3.21) follows from (3.5). It remains to prove (3.23). We set $L_{k}^{\prime}=\sum_{i=\lceil a t\rceil}^{k} \mathbb{I}_{i} U_{i}+\sum_{i=k+1}^{\lfloor b t\rfloor} \eta_{i} U_{i}$. We have, by the triangle inequality,

$$
\tilde{d}_{T V}\left(L_{t}, L_{0 t}\right) \leq \sum_{k \in T_{t}} \tilde{d}_{T V}\left(L_{k-1}^{\prime}, L_{k}^{\prime}\right) .
$$

Then we estimate $\tilde{d}_{T V}\left(L_{k-1}^{\prime}, L_{k}^{\prime}\right) \leq \tilde{d}_{T V}\left(\eta_{k}, \mathbb{I}_{k}\right) \leq(k t)^{-1} Y_{t}^{2} X_{k}^{2}$. Here the first inequality follows from the properties of the total variation distance. The second inequality follows from Lemma 3.2 and the fact that on the event $\mathcal{A}_{t}$ we have $p_{k t}=\lambda_{k t}$.

Let us prove the second bound of (3.21). In view of (3.4) it suffices to show that $\tilde{d}_{T V}\left(L_{0 t}, L_{1 t}\right)=o_{P}(1)$. For this purpose we write, by the triangle inequality,

$$
\tilde{d}_{T V}\left(L_{0 t}, L_{1 t}\right) \leq \sum_{k \in T_{t}} \tilde{d}_{T V}\left(L_{k-1}^{*}, L_{k}^{*}\right)
$$

where $L_{k}^{*}:=\sum_{i=\lceil a t\rceil}^{k} \eta_{i} U_{i}+\sum_{i=k+1}^{\lfloor b t\rfloor} \eta_{i} \xi_{1 i}$, and estimate

$$
\tilde{d}_{T V}\left(L_{k-1}^{*}, L_{k}^{*}\right) \leq \tilde{d}_{T V}\left(\eta_{k} U_{k}, \eta_{k} \xi_{1 k}\right) \leq \tilde{\mathbf{P}}\left(\eta_{k} \neq 0\right) \tilde{d}_{T V}\left(U_{k}, \xi_{1 k}\right) .
$$

Now, invoking the inequalities

$$
\tilde{\mathbf{P}}\left(\eta_{k} \neq 0\right)=1-e^{-\lambda_{k t}} \leq \lambda_{k t} \quad \text { and } \quad \tilde{d}_{T V}\left(U_{k}, \xi_{1 k}\right) \leq \sum_{j \in T_{k}^{*} \backslash\{t\}} p_{k j}^{2},
$$

see (3.19), we obtain from (3.24), (3.25) and (3.11) that

$$
\tilde{d}_{T V}\left(L_{0 t}, L_{1 t}\right) \leq Q_{X Y}(t)=o_{P}(1) .
$$

Let us prove the first bound of (3.22). We observe that

$$
\left|L_{2 t}-L_{1 t}\right|=L_{2 t}-L_{1 t}=\sum_{i \in T_{t}} \eta_{i} \Delta_{1 i}
$$

and

$$
\tilde{\mathbf{E}} \sum_{i \in T_{t}} \eta_{i} \Delta_{1 i}=\sum_{i \in T_{t}} \lambda_{i t} \sum_{j \in T_{i}^{*} \backslash\{t\}}\left(\lambda_{i j}-1\right) \mathbb{I}_{\left\{\lambda_{i j}>1\right\}} \leq Q_{X Y}(t) .
$$

We obtain $\mathbf{E}\left|L_{2 t}-L_{1 t}\right| \leq \mathbf{E} Q_{X Y}(t)=o(1)$, see (3.11).

Let us prove the second bound of (3.22). We note that the random vector

$$
\left(\tilde{L}_{2 t}, \tilde{L}_{3 t}\right), \quad \tilde{L}_{2 t}=\sum_{i \in T_{t}} \eta_{i}\left(\xi_{4 i}+\Delta_{2 i}\right), \quad \tilde{L}_{3 t}=\sum_{i \in T_{t}} \eta_{i}\left(\xi_{4 i}+\Delta_{3 i}\right)
$$


has the marginal distributions of $\left(L_{2 t}, L_{3 t}\right)$. In addition, since $\Delta_{2 i}, \Delta_{3 i} \geq 0$ and at most one of them is nonzero, we have $\left|\Delta_{2 i}-\Delta_{3 i}\right|=\Delta_{2 i}+\Delta_{3 i}$. Therefore, we can write

$$
\tilde{\Delta}:=\left|\tilde{L}_{2 t}-\tilde{L}_{3 t}\right| \leq \sum_{i \in T_{t}}\left|\eta_{i}\right|\left|\Delta_{2 i}-\Delta_{3 i}\right|=\sum_{i \in T_{t}} \eta_{i}\left(\Delta_{2 i}+\Delta_{3 i}\right) .
$$

We remark that given $X, Y$, the random variable $\Delta_{2 i}+\Delta_{3 i}$ has Poisson distribution with (conditional) mean value

$$
\tilde{\mathbf{E}}\left(\Delta_{2 i}+\Delta_{3 i}\right)=X_{i} i^{-1 / 2} \delta_{i}, \quad \delta_{i}:=\left|B_{1}\left(T_{i}^{*} \backslash\{t\}\right)-b_{1} \gamma_{2} i^{1 / 2}\right| .
$$

Therefore, (3.27) implies $\tilde{\mathbf{E}} \tilde{\Delta} \leq t^{-1 / 2} Y_{t} \sum_{i \in T_{t}} X_{i}^{2} i^{-1} \delta_{i}$. Next, for $0<\varepsilon<1$, we have

$$
\mathbf{E} \mathbb{I}_{\mathcal{B}_{t}(\varepsilon)} \tilde{\Delta} \leq \mathbf{E I}_{\mathcal{B}_{t}(\varepsilon)} t^{-1 / 2} Y_{t} \sum_{i \in T_{t}} X_{i}^{2} i^{-1} \delta_{i}=b_{1} a_{2} t^{-1 / 2} \sum_{i \in T_{t}} i^{-1} \mathbf{E} \delta_{i} \mathbb{I}_{\mathcal{B}_{t}(\varepsilon)} .
$$

Invoking upper bound (3.13) for $\mathbf{E} \delta_{i} \mathbb{I}_{\mathcal{B}_{t}(\varepsilon)}$, we obtain $\mathbf{E} \mathbb{I}_{\mathcal{B}_{t}(\varepsilon)} \tilde{\Delta} \leq c b_{1}^{3 / 2} a_{2} \varepsilon+o(1)$. Finally, this bound combined with Markov's inequality and (3.7) yields

$$
\mathbf{P}(\tilde{\Delta} \geq 1)=\mathbf{P}\left(\{\tilde{\Delta} \geq 1\} \cap \mathcal{B}_{t}(\varepsilon)\right)+o(1) \leq \mathbf{E I}_{\mathcal{B}_{t}(\varepsilon)} \tilde{\Delta}+o(1) \leq c b_{1}^{3 / 2} a_{2} \varepsilon+o(1) .
$$

We conclude that $\mathbf{P}(\tilde{\Delta} \neq 0)=\mathbf{P}(\tilde{\Delta} \geq 1)=o(1)$, thus obtaining the second bound of (3.22).

Step 2. Here we prove that $L_{3 t}$ converges in distribution to $d_{*}$. Before the proof, we introduce some notation. Let $Y_{\star}$ be a random variable with the same distribution as $Y_{1}$ and independent of $X, Y$. Given $X, Y, Y_{\star}$, we generate independent Poisson random variables $\eta_{k}^{\star}, k \in T_{t}$ with (conditional) mean values $\mathbf{E}\left(\eta_{k}^{\star} \mid X, Y, Y_{\star}\right)=\lambda_{k \star}$, where $\lambda_{k \star}=X_{k} Y_{\star}(k t)^{-1 / 2}$. We assume that, given $X, Y, Y_{\star}$, the family of random variables $\left\{\eta_{k}^{\star}, k \in T_{t}\right\}$ is conditionally independent of $\left\{\xi_{3 k}, k \in T_{t}\right\}$. Define $L_{t}^{\star}=\sum_{k \in T_{t}} \eta_{k}^{\star} \xi_{3 k}$, and let $d_{\star}$ be defined in the same way as $d_{*}$, but with $\lambda_{1}$ replaced by $\lambda_{\star}=Y_{\star} a_{1} \gamma_{1}$. Denote

$$
\begin{gathered}
f_{\varkappa}(z)=\mathbf{E} e^{i z \varkappa_{1}}, \quad \bar{f}_{\varkappa}(z)=\sum_{r \geq 0} e^{i z r} \bar{p}_{r}, \quad \bar{p}_{r}=\bar{\lambda}^{-1} \sum_{k \in T_{t}} \lambda_{k \star} \mathbb{I}_{\left\{\xi_{3 k}=r\right\}}, \quad \bar{\lambda}=\sum_{k \in T_{t}} \lambda_{k \star}, \\
\delta=\left(\bar{f}_{\varkappa}(z)-1\right) \bar{\lambda}-\left(f_{\varkappa}(z)-1\right) \lambda_{\star}, \quad f(z)=\overline{\mathbf{E}} e^{i z d_{\star}}, \quad \bar{f}(z)=\overline{\mathbf{E}} e^{i z L_{t}^{\star}} .
\end{gathered}
$$

Here and following, $\boldsymbol{i}$ denotes the imaginary unit, $z$ is a real number. $\overline{\mathbf{E}}$ denotes the conditional expectation given $X, Y, Y_{\star}$ and $\left\{\xi_{3 k}, k \in T_{t}\right\}$. By $\mathbf{E}_{\star}$ we denote the conditional expectation given $Y_{\star}$. We recall that random variables $\varkappa_{1}, \varkappa_{2}, \ldots$ are defined in the statement of Theorem 2.1. Given $\varepsilon>0$, introduce the event $\mathcal{D}=\mathcal{D}_{\varepsilon}=\left\{\left|A_{1}\left(T_{t}\right)-\gamma_{1} a_{1} t^{1 / 2}\right|<\right.$ $\left.\varepsilon t^{1 / 2} \min \left\{1, \gamma_{1} a_{1}\right\}\right\}$ and let $\overline{\mathcal{D}}$ denote the complement event. Furthermore, select the number $T=T_{\varepsilon}>1 / \varepsilon$ such that $\mathbf{P}\left(\varkappa_{1} \geq T\right)<\varepsilon$.

We note that $L_{t}^{\star}$ is defined in the same way as $L_{3 t}$ above, but with $Y_{t}$ replaced by $Y_{\star}$. Hence $L_{3 t}$ has the same distribution as $L_{t}^{\star}$. Similarly, $d_{*}$ has the same distribution as $d_{\star}$. Therefore, it suffices to show that $L_{t}^{\star}$ converges in distribution to $d_{\star}$. For this purpose, we show the convergence of Fourier-Stieltjes transforms $\mathbf{E} e^{i z L_{t}^{\star}} \rightarrow \mathbf{E} e^{i z d_{\star}}$. We observe that, given $Y_{\star}$, the conditional distribution of $d_{\star}$ is the compound Poisson distribution with the characteristic function $f(z)=e^{\lambda_{\star}\left(f_{\varkappa}(z)-1\right)}$. Similarly, given $X, Y, Y_{\star}$ and $\left\{\xi_{3 k}, k \in T_{t}\right\}$, the conditional distribution of $L_{t}^{\star}$ is the compound Poisson distribution with the characteristic function $\bar{f}(z)=e^{\bar{\lambda}\left(\bar{f}_{\varkappa}(z)-1\right)}$. In the proof we exploit the convergence $\bar{\lambda} \rightarrow \lambda_{\star}$ and $\bar{f}_{\varkappa}(z) \rightarrow$ 
$f_{\varkappa}(z)$. Denote $\Delta^{\star}(z)=e^{i z L_{t}^{\star}}-e^{i z d_{\star}}$. We shall show below that, for any real $z$ and any realized value $Y_{\star}$ there exists a positive constant $c^{\star}=c^{\star}\left(z, Y_{\star}\right)$ such that for every $0<\varepsilon<0.5$ we have

$$
\limsup _{t}\left|\mathbf{E}\left(\Delta^{\star}(z) \mid Y_{\star}\right)\right|<c^{\star} \varepsilon
$$

We remark that (3.28) implies $\mathbf{E}\left(\Delta^{\star}(z) \mid Y_{\star}\right)=o(1)$. This fact together with the simple inequality $\left|\Delta^{\star}(z)\right| \leq 2$ yields $\mathbf{E} \Delta^{\star}(z)=o(1)$, by Lebesgue's dominated convergence theorem. Finally, the identity $\mathbf{E} \Delta^{\star}(z)=\mathbf{E} e^{i z L_{t}^{\star}}-\mathbf{E} e^{i z d_{\star}}$ implies the desired convergence $\mathbf{E} e^{i z L_{t}^{\star}} \rightarrow \mathbf{E} e^{i z d_{\star}}$ as $t \rightarrow+\infty$.

Let us fix $0<\varepsilon<0.5$ and prove (3.28). We write

$$
\mathbf{E}_{\star} \Delta^{\star}(z)=I_{1}+I_{2}, \quad I_{1}=\mathbf{E}_{\star} \Delta^{\star}(z) \mathbb{I}_{\mathcal{D}}, \quad I_{2}=\mathbf{E}_{\star} \Delta^{\star}(z) \mathbb{I}_{\overline{\mathcal{D}}},
$$

and observe that $\left|I_{2}\right| \leq 2 \mathbf{P}_{\star}(\overline{\mathcal{D}})=2 \mathbf{P}(\overline{\mathcal{D}})=o(1)$. Indeed, the bound $\mathbf{P}(\overline{\mathcal{D}})=o(1)$ follows from (3.14), by Markov's inequality. Let us estimate $I_{1}$. Combining the identity $\overline{\mathbf{E}} \Delta^{\star}(z)=\overline{\mathbf{E}} f(z)\left(e^{\delta}-1\right)$ with the inequalities $|f(z)| \leq 1$ and $\left|e^{s}-1\right| \leq|s| e^{|s|}$, we obtain

$$
\left|I_{1}\right| \leq \mathbf{E}_{\star}|\delta| e^{|\delta|} \mathbb{I}_{\mathcal{D}} \leq c_{1}^{\star} \mathbf{E}_{\star}|\delta| \mathbb{I}_{\mathcal{D}}
$$

Here we estimated $e^{|\delta|} \leq e^{6 \lambda_{\star}}=: c_{1}^{\star}$ using the inequalities

$$
|\delta| \leq 2 \bar{\lambda}+2 \lambda_{\star}, \quad \bar{\lambda}=Y_{\star} t^{-1 / 2} A_{1}\left(T_{t}\right) \leq 2 \lambda_{\star} .
$$

We remark that the last inequality holds, provided that event $\mathcal{D}$ occurs. Here and in what follows $c_{1}^{\star}, c_{2}^{\star}, \ldots$ denote positive numbers that do not depend on $t$.

We complete the proof of (3.28) by showing that $\mathbf{E}_{\star}|\delta| \mathbb{I}_{\mathcal{D}} \leq\left(c_{2}^{\star}+c_{3}^{\star} \lambda_{\star}+c_{4}^{\star} \lambda_{\star}\right) \varepsilon+$ $o(1)$, see also (3.29), (3.30). To this aim we write

$$
\delta=\left(\bar{f}_{\varkappa}(z)-1\right)\left(\bar{\lambda}-\lambda_{\star}\right)+\left(\bar{f}_{\varkappa}(z)-f_{\varkappa}(z)\right) \lambda_{\star}
$$

and estimate $|\delta| \leq 2\left|\bar{\lambda}-\lambda_{\star}\right|+\lambda_{\star}\left|\bar{f}_{\varkappa}(z)-f_{\varkappa}(z)\right|$. The inequality, which holds on the event $\mathcal{D},\left|\bar{\lambda}-\lambda_{\star}\right| \leq Y_{\star} \varepsilon$ implies $\mathbf{E}_{\star}\left|\bar{\lambda}-\lambda_{\star}\right| \mathbb{I}_{\mathcal{D}} \leq c_{2}^{\star} \varepsilon$ with $c_{2}^{\star}:=Y_{\star}$. It remains to show that

$$
\mathbf{E}_{\star}\left|\bar{f}_{\varkappa}(z)-f_{\varkappa}(z)\right| \mathbb{I}_{\mathcal{D}} \leq\left(c_{3}^{\star}+c_{4}^{\star}\right) \varepsilon+o(1) .
$$

We split

$$
\bar{f}_{\varkappa}(z)-f_{\varkappa}(z)=\sum_{r \geq 0} e^{i z r}\left(\bar{p}_{r}-p_{r}\right)=R_{1}-R_{2}+R_{3}
$$

and estimate separately the terms

$$
R_{1}=\sum_{r \geq T} e^{i z r} \bar{p}_{r}, \quad R_{2}=\sum_{r \geq T} e^{i z r} p_{r}, \quad R_{3}=\sum_{0 \leq r<T} e^{i z r}\left(\bar{p}_{r}-p_{r}\right) .
$$

Here we denote $p_{r}=\mathbf{P}\left(\varkappa_{1}=r\right)$.

The upper bound for $R_{2}$ follows by the choice of $T$

$$
\left|R_{2}\right| \leq \sum_{r \geq T} p_{r}=\mathbf{P}\left(\varkappa_{1} \geq T\right)<\varepsilon .
$$


Next, combining the identities $\bar{p}_{r}=\left(A_{1}\left(T_{t}\right)\right)^{-1} \sum_{k \in T_{t}} k^{-1 / 2} X_{k} \mathbb{I}_{\left\{\xi_{3 k}=r\right\}}$ and

$$
\sum_{r \geq T} \sum_{k \in T_{t}} k^{-1 / 2} X_{k} \mathbb{I}_{\left\{\xi_{3 k}=r\right\}}=\sum_{k \in T_{t}} k^{-1 / 2} X_{k} \mathbb{I}_{\left\{\xi_{3 k} \geq T\right\}}
$$

with the inequality $A_{1}\left(T_{t}\right) \geq t^{1 / 2} a_{1} \gamma_{1} / 2$, which holds on the event $\mathcal{D}$, we obtain

$$
\left|R_{1}\right| \mathbb{I}_{\mathcal{D}} \leq \sum_{r \geq T} \bar{p}_{r} \leq \frac{2}{a_{1} \gamma_{1} t^{1 / 2}} \sum_{k \in T_{t}} \frac{X_{k}}{k^{1 / 2}} \mathbb{I}_{\left\{\xi_{3 k} \geq T\right\}} \leq \frac{2}{a_{1} \gamma_{1} t^{1 / 2}} \sum_{k \in T_{t}} \frac{X_{k} \xi_{3 k}}{T k^{1 / 2}} .
$$

Now, the identity $\mathbf{E}_{\star} X_{k} \xi_{3 k}=a_{2} b_{1} \gamma_{2}$ implies $\mathbf{E}_{\star}\left|R_{1}\right| \mathbb{I}_{\mathcal{D}} \leq c_{4}^{\star} T^{-1} \leq c_{4}^{\star} \varepsilon$.

Let us estimate $R_{3}$. We denote $p_{r}^{\prime}=A_{1}\left(T_{t}\right)\left(a_{1} \gamma_{1} t^{1 / 2}\right)^{-1} \bar{p}_{r}$ and observe that the inequality $\left|A_{1}\left(T_{t}\right)\left(a_{1} \gamma_{1} t^{1 / 2}\right)^{-1}-1\right| \leq \varepsilon$, which holds on the event $\mathcal{D}$, implies

$$
\left|\sum_{0 \leq r \leq T} e^{i t r}\left(\bar{p}_{r}-p_{r}^{\prime}\right)\right| \mathbb{I}_{\mathcal{D}} \leq \varepsilon \sum_{0 \leq r \leq T} \bar{p}_{r} \leq \varepsilon .
$$

In the last inequality we use the fact that the probabilities $\left\{\bar{p}_{r}\right\}_{r \geq 0}$ sum to 1 . It follows now that

$$
\left|R_{3}\right| \mathbb{I}_{\mathcal{D}} \leq \varepsilon+\sum_{0 \leq r \leq T}\left|p_{r}^{\prime}-p_{r}\right|
$$

Next we estimate

$$
\mathbf{E}_{\star}\left|p_{r}^{\prime}-p_{r}\right| \leq \mathbf{E}_{\star}\left|p_{r}^{\prime}-\mathbf{E}_{\star} p_{r}^{\prime}\right|+\left|\mathbf{E}_{\star} p_{r}^{\prime}-p_{r}\right|
$$

and

$$
\begin{aligned}
& \left(\mathbf{E}_{\star}\left|p_{r}^{\prime}-\mathbf{E}_{\star} p_{r}^{\prime}\right|\right)^{2} \leq \mathbf{E}_{\star}\left|p_{r}^{\prime}-\mathbf{E}_{\star} p_{r}^{\prime}\right|^{2} \leq\left(a_{1} \gamma_{1} t^{1 / 2}\right)^{-2} \sum_{k \in T_{t}} k^{-1} a_{2} \leq c t^{-1} a_{2} a_{1}^{-2}, \\
& \left|\mathbf{E}_{\star} p_{r}^{\prime}-p_{r}\right|=p_{r}\left|1-\left(\gamma_{1} t^{1 / 2}\right)^{-1} \sum_{k \in T_{t}} k^{-1 / 2}\right| \leq c t^{-1} .
\end{aligned}
$$

In (3.33) we first apply the Cauchy-Schwartz inequality, then the linearity of variance of a sum of independent random variables, and then the inequality $\operatorname{Var} X_{k} \mathbb{I}_{\left\{\xi_{3 k}=r\right\}} \leq$ $\mathbf{E}\left(X_{k} \mathbb{I}_{\left\{\xi_{3 k}=r\right\}}\right)^{2} \leq a_{2}$. In (3.34) we use the identity $\mathbf{E}_{\star} X_{k} \mathbb{I}_{\left\{\xi_{3 k}=r\right\}}=a_{1} p_{r}$ and (3.6). From (3.32), (3.33), (3.34) we conclude that $\mathbf{E}_{\star}\left|p_{r}^{\prime}-p_{r}\right|=O\left(t^{-1 / 2}\right)$. Finally, (3.31) implies

$$
\mathbf{E}_{\star}\left|R_{3}\right| \mathbb{I}_{\mathcal{D}} \leq \varepsilon+O\left(|T| t^{-1 / 2}\right)=\varepsilon+o(1) .
$$




\subsection{Proof of Theorem 2.2}

Here, we assume that $\tau(t)=t^{\nu}, v>1$. In the proof, we apply the following simple approximations

$$
\begin{array}{rlrl}
\sum_{k \in T_{t}} k^{-(1-2 v) /(2 v)} & =t^{1 / 2} \gamma_{1}^{\prime}+r t^{(2 v)^{-1}-1}, & \sum_{j \in T_{k}^{*}} j^{-1 / 2} & =k^{(2 v)^{-1}} \gamma_{2}^{\prime}+r^{\prime} k^{-(2 v)^{-1}}, \\
\gamma_{1}^{\prime}:=2 v\left(b^{(2 v)^{-1}}-a^{(2 v)^{-1}}\right), & \gamma_{2}^{\prime}:=2\left(a^{-(2 v)^{-1}}-b^{-(2 v)^{-1}}\right),
\end{array}
$$

where $|r|,\left|r^{\prime}\right| \leq c$. We also make use of relations (3.7), (3.9), (3.10) and (3.11), which remain valid in the case where $\tau(t)=t^{v}$, and of the inequalities, for $k \in T_{t}$,

$$
\begin{aligned}
& \left|\mathbf{E} B_{1}\left(T_{k}^{*} \backslash\{t\}\right)-b_{1} \gamma_{2}^{\prime} k^{1 /(2 v)}\right| \leq c b_{1} k^{-1 /(2 v)}, \\
& \mathbf{E}\left|B_{1}\left(T_{k}^{*} \backslash\{t\}\right)-b_{1} \gamma_{2}^{\prime} k^{1 /(2 v)}\right| \mathbb{I}_{\mathcal{B}_{t}(\varepsilon)} \leq c k^{1 /(2 v)}\left(\varepsilon b_{1}^{1 / 2}+\mathbf{E} Y_{1} \mathbb{I}_{\left\{Y_{1}>\varepsilon^{2} t_{-}\right\}}\right)+c b_{1} k^{-1 /(2 v)} .
\end{aligned}
$$

We note that (3.36) follows from the second identity of (3.35), and (3.37) is obtained in the same way as (3.13).

Proof of Theorem 2.2. Before the proof we introduce some notation. Given $\varepsilon \in(0,1)$, denote

$$
\zeta=\sum_{k \in T_{t}} \lambda_{k t} \zeta_{k}, \quad \zeta_{k}=\beta_{k} b_{1} X_{k} \mathbb{I}_{k}^{\prime}, \quad \mathbb{I}_{k}^{\prime}=\mathbb{I}_{\left\{\beta_{k} b_{1} X_{k}<\varepsilon\right\}}, \quad \beta_{k}=k^{(1-v) /(2 v)} \gamma_{2}^{\prime}
$$

Given $X, Y$, we generate independent Poisson random variables $\eta_{k}, \hat{\xi}_{3 k}, k \in T_{t}$, with (conditional) mean values $\tilde{\mathbf{E}} \eta_{k}=\lambda_{k t}, \tilde{\mathbf{E}} \hat{\xi}_{3 k}=\beta_{k} b_{1} X_{k}$. We also generate independent Bernoulli random variables $\tilde{\mathbb{I}}_{k}, k \in T_{t}$ with success probabilities

$$
\tilde{\mathbf{P}}\left(\tilde{\mathbb{I}}_{k}=1\right)=1-\tilde{\mathbf{P}}\left(\tilde{\mathbb{I}}_{k}=0\right)=\zeta_{k}
$$

and introduce random variables

$$
\hat{L}_{3 t}=\sum_{k \in T_{t}} \eta_{k} \hat{\xi}_{3 k}, \quad L_{4 t}=\sum_{k \in T_{t}} \mathbb{I}_{k} \hat{\xi}_{3 k}, \quad L_{5 t}=\sum_{k \in T_{t}} \mathbb{I}_{k} \mathbb{I}_{k}^{\prime} \hat{\xi}_{3 k}, \quad L_{6 t}=\sum_{k \in T_{t}} \mathbb{I}_{k} \tilde{\mathbb{I}}_{k} .
$$

We assume that, given $X, Y$, the sequences $\left\{\mathbb{I}_{k}, k \in T_{t}\right\},\left\{\tilde{\mathbb{I}}_{k}, k \in T_{t}\right\},\left\{\eta_{k}, k \in T_{t}\right\},\left\{\hat{\xi}_{3 k}, k \in\right.$ $\left.T_{t}\right\}$ are conditionally independent. Furthermore, we define the random variable $L_{7 t}$ as follows. We first generate $X, Y$. Then, given $X, Y$, we generate a Poisson random variable with the conditional mean value $\zeta$. The realized value of the Poisson random variable is denoted $L_{7 t}$. Thus, we have $\mathbf{P}\left(L_{7 t}=r\right)=\mathbf{E} e^{-\zeta} \zeta^{r} / r$ !, for $r=0,1, \ldots$

Now we are ready to prove Theorem 2.2. Proceeding in the same way as in the proof of (3.21), (3.22) above (and making use of (3.7), (3.9), (3.10), (3.11), (3.36), (3.37)) we show that the random variables $d\left(v_{t}\right)$ and $\hat{L}_{3 t}$ have the same asymptotic distribution (if any). It remains to show that $\hat{L}_{3 t}$ converges in distribution to $\Lambda_{3}$. For this purpose we prove that

$$
d_{T V}\left(\hat{L}_{3 t}, L_{4 t}\right)=o(1), \quad \mathbf{E}\left(L_{4 t}-L_{5 t}\right)=o(1), \quad d_{T V}\left(L_{6 t}, L_{7 t}\right)=o(1)
$$

and there exists $c>0$, depending only on $a, b, v$, such that 


$$
d_{T V}\left(L_{5 t}, L_{6 t}\right) \leq c a_{2} b_{1}^{2} \varepsilon
$$

Furthermore, for every real $z$,

$$
\mathbf{E} e^{i z L_{7 t}}-\mathbf{E} e^{i z \Lambda_{3}}=o(1)
$$

(here $i$ denotes the imaginary unit). Clearly, from (3.38), (3.40), (3.39) we obtain that $\hat{L}_{3 t}$ converges in distribution to $\Lambda_{3}$.

Let us prove (3.38), (3.39), (3.40). The first bound of (3.38) is obtained in the same way as that of (3.21). To show the second bound of (3.38) we write

$$
\tilde{\mathbf{E}}\left(L_{4 t}-L_{5 t}\right)=\sum_{k \in T_{t}}\left(1-\mathbb{I}_{k}^{\prime}\right) \tilde{\mathbf{E}} \mathbb{I}_{k} \tilde{\mathbf{E}} \hat{\xi}_{3 k}=Y_{t} b_{1} t^{-1 / 2} \sum_{k \in T_{t}}\left(1-\mathbb{I}_{k}^{\prime}\right) X_{k}^{2} \beta_{k} k^{-1 / 2}
$$

and apply the inequality

$$
\mathbf{E} X_{k}^{2}\left(1-\mathbb{I}_{k}^{\prime}\right) \leq \mathbf{E} X_{\underline{t}}^{2}\left(1-\mathbb{I}_{\underline{t}}^{\prime}\right), \quad k \in T_{t} .
$$

where $\underline{t}=\min \left\{k: k \in T_{t}\right\}$. In this way we obtain

$$
\mathbf{E}\left(L_{4 t}-L_{5 t}\right)=\mathbf{E} \tilde{\mathbf{E}}\left(L_{4 t}-L_{5 t}\right) \leq S_{t} b_{1}^{2} \mathbf{E} X_{\underline{t}}^{2}\left(1-\mathbb{I}_{\underline{t}}^{\prime}\right)=o(1) .
$$

Here $S_{t}=t^{-1 / 2} \sum_{k \in T_{t}} \beta_{k} k^{-1 / 2}$ is uniformly bounded, and $\mathbf{E} X_{\underline{t}}^{2}\left(1-\mathbb{I}_{\underline{t}}^{\prime}\right)=o(1)$, since $\underline{t} \rightarrow+\infty$ as $t \rightarrow+\infty$.

Let us prove the third bound of (3.38). In view of (3.4) it suffices to show that $\tilde{d}_{T V}\left(L_{6 t}, L_{7 t}\right)=o_{P}(1)$. For this purpose we write

$$
\tilde{d}_{T V}\left(L_{6 t}, L_{7 t}\right) \leq \mathbb{I}_{\mathcal{A}_{t}} \tilde{d}_{T V}\left(L_{6 t}, L_{7 t}\right)+\mathbb{I}_{\overline{\mathcal{A}}_{t}},
$$

where $\mathbb{I}_{\overline{\mathcal{A}}_{t}}=o_{P}(1)$, see (3.10), and estimate using Le Cam's inequality (3.19)

$$
\mathbb{I}_{\mathcal{A}_{t}} \tilde{d}_{T V}\left(L_{6 t}, L_{7 t}\right) \leq \mathbb{I}_{\mathcal{A}_{t}} \sum_{k \in T_{t}} \tilde{\mathbf{P}}^{2}\left(\mathbb{I}_{k} \tilde{\mathbb{I}}_{k}=1\right) \mathbb{I}_{k}^{\prime} \leq Y_{t}^{2} b_{1}^{2} t^{-1} \sum_{k \in T_{t}} k^{-1} \beta_{k}^{2} X_{k}^{4}=o_{P}(1)
$$

Here we used the simple inequality $t^{-1} \sum_{k \in T_{t}} k^{-1} \beta_{k}^{2} X_{k}^{4} \leq c t^{-2 v} \sum_{k \leq b t^{v}} X_{k}^{4}$ and the fact that $\mathbf{E} X_{1}^{2}<\infty$ implies the bound $n^{-2} \sum_{k \leq n} X_{k}^{4}=o_{P}(1)$, as $n \rightarrow+\infty$.

Let us prove (3.39). Proceeding as in (3.24), (3.25) and using the identity $\tilde{\mathbb{I}}_{k}=\tilde{\mathbb{I}}_{k} \mathbb{I}_{k}^{\prime}$ we have

$$
\tilde{d}_{T V}\left(L_{5 t}, L_{6 t}\right) \leq \sum_{k \in T_{t}} \mathbb{I}_{k}^{\prime} \tilde{\mathbf{P}}\left(\mathbb{I}_{k} \neq 0\right) \tilde{d}_{T V}\left(\hat{\xi}_{3 k}, \tilde{\mathbb{I}}_{k}\right)
$$

Next, we estimate $\mathbb{I}_{k}^{\prime} \tilde{d}_{T V}\left(\hat{\xi}_{3 k}, \tilde{\mathbb{I}}_{k}\right) \leq \zeta_{k}^{2}$, by Le Cam's inequality (3.19), and invoke the inequality $\tilde{\mathbf{P}}\left(\mathbb{I}_{k} \neq 0\right) \leq \lambda_{k t}$. We obtain

$$
\tilde{d}_{T V}\left(L_{5 t}, L_{6 t}\right) \leq \sum_{k \in T_{t}} \mathbb{I}_{k}^{\prime} \lambda_{k t} \zeta_{k}^{2} \leq \varepsilon \sum_{k \in T_{t}} \lambda_{k t} \zeta_{k}
$$


Here we estimated $\zeta_{k}^{2} \leq \varepsilon \zeta_{k}$. Now, the inequalities

$$
d_{T V}\left(L_{5 t}, L_{6 t}\right) \leq \mathbf{E} \tilde{d}_{T V}\left(L_{5 t}, L_{6 t}\right) \leq \varepsilon \sum_{k \in T_{t}} \mathbf{E} \lambda_{k t} \zeta_{k} \leq a_{2} b_{1}^{2} S_{t} \varepsilon
$$

and the fact that $S_{t}$ is uniformly bounded, i.e., $S_{t} \leq c$, for some $c>0$, imply (3.39).

Finally, we show (3.40). We write $\tilde{\mathbf{E}} e^{i z L_{7 t}}=e^{\zeta\left(e^{i z}-1\right)}$ and use the bound

$$
Y_{t} b_{1} a_{2} \gamma-\zeta=o_{P}(1)
$$

We note that since the function $u \rightarrow e^{u\left(e^{i z}-1\right)}$ is bounded and uniformly continuous, for $u \geq 0$, relation (3.42) implies the convergence

$$
\mathbf{E} e^{i z L_{7 t}}=\mathbf{E} e^{\zeta\left(e^{i z}-1\right)} \rightarrow \mathbf{E} e^{Y_{t} b_{1} a_{2} \gamma\left(e^{i z}-1\right)}=\mathbf{E} e^{i z \Lambda_{3}} .
$$

It remains to prove (3.42). Proceeding as in the proof of the second bound of (3.38) we obtain

$$
\zeta=Y_{t} b_{1} \gamma_{2}^{\prime} t^{-1 / 2} \sum_{k \in T_{t}} X_{k}^{2} k^{(2 v)^{-1}-1}+o p(1)
$$

Then we split $\gamma=\gamma_{1}^{\prime} \gamma_{2}^{\prime}$ and invoke the expression for $\gamma_{1}^{\prime}$ obtained from (3.35). We obtain

$$
Y_{t} b_{1} a_{2} \gamma=Y_{t} b_{1} \gamma_{2}^{\prime} t^{-1 / 2} \sum_{k \in T_{t}} k^{-(1-2 v) /(2 v)} a_{2}+o_{P}(1) .
$$

Now, we observe that (3.42) follows from (3.43), (3.44) and the bound

$$
t^{-1 / 2} \sum_{k \in T_{t}}\left(a_{2}-X_{k}^{2}\right) k^{(2 v)^{-1}-1}=o_{P}(1)
$$

which is obtained from Chebyshev's inequality using a truncation argument.

\section{FUNDING}

Research of M. Bloznelis was supported by the Research Council of Lithuania grant MIP067/2013.

\section{REFERENCES}

[1] W. Aiello, A. Bonato, C. Cooper, J. Janssen, and P. Pralat. "A Spatial Web Graph Model with Local Influence Regions." In Proceedings of the 5th Workshop on Algorithms and Models for the Web-Graph (WAW2007), edited by A. Bonato, and F. R. K. Chung, pp. 96-107. LNCS 4863. Berlin, Heidelberg: Springer, 2007.

[2] M. Bloznelis. "Degree and Clustering Coefficient in Sparse Random Intersection Graphs." The Annals of Applied Probability 23 (2013), 1254-1289.

[3] M. Bloznelis and J. Damarackas. "Degree Distribution of an Inhomogeneous Random Intersection Graph." The Electronic Journal of Combinatorics 20:3 (2013), R3.

[4] M. Bloznelis and F. Götze. "Preferred Attachment Model of Affiliation Network." Journal of Statistical Physics 156 (2014), 800-821. 
[5] M. Bloznelis, J. Jaworski, and V. Kurauskas. "Assortativity and Clustering of Sparse Random Intersection Graphs.” Electronic Journal of Probability 18 (2013), R38.

[6] M. Bloznelis and V. Kurauskas. "Clustering Function: A Measure of Social Influence." Journal of Complex Networks (2015) doi:10.1093/comnet/cnv010

[7] M. Bloznelis and M. Karoński. "Random Intersection Graph Process." In WAW 2013, edited by A. Bonato, M. Mitzenmacher, and P. Pralat, pp. 93-105, LNCS 8305. Berlin, Heidelberg: Springer, 2013.

[8] M. Deijfen, and W. Kets. "Random Intersection Graphs with Tunable Degree Distribution and Clustering." Probab. Engrg. Inform. Sci. 23 (2009), 661-674.

[9] S. Foss, D. Korshunov, and S. Zachary. "An Introduction to Heavy-Tailed and Subexponential Distributions.” New York, NY, USA: ACM, 2011.

[10] E. Godehardt and J. Jaworski. "Two Models of Random Intersection Graphs and Their Applications." Electronic Notes in Discrete Mathematics 10 (2001), 129-132.

[11] E. Godehardt, J. Jaworski, and K. Rybarczyk. "Clustering Coefficients of Random Intersection Graphs." In Studies in Classification, Data Analysis and Knowledge Organization, pp. 243-253. Berlin, Heidelberg: Springer, 2012.

[12] J. L. Guillaume and M. Latapy. "Bipartite Structure of all Complex Networks." Inform. Process. Lett. 90 (2004), 215-221.

[13] J. Jaworski, M. Karoński, and D. Stark. "The Degree of a Typical Vertex in Generalized Random Intersection Graph Models.” Discrete Mathematics 306 (2006), 2152-2165.

[14] M. Karoński, E. R. Scheinerman, and K.B. Singer-Cohen. "On Random Intersection Graphs: The Subgraph Problem." Combinatorics, Probability and Computing 8 (1999), 131-159.

[15] T. Martin, B. Ball, B. Karrer, M. E. J. Newman. "Coauthorship and Citation Patterns in the Physical Review." Phys. Rev. E 88 (2013), 012814.

[16] M. E. J. Newman. "Clustering and Preferential Attachment in Growing Networks." Physical Review E 64 (2001), 025102.

[17] M. E. J. Newman, D. J. Watts, S. H. Strogatz. "Random Graph Models of Social Networks." Proc. Natl. Acad. Sci. USA, 99:Suppl. 1 (2002), 2566-2572.

[18] D. Stark. "The Vertex Degree Distribution of Random Intersection Graphs.” Random Structures and Algorithms 24 (2004), 249-258.

[19] J. M. Steele. "Le Cam's Inequality and Poisson Approximations." The American Mathematical Monthly 101 (1994), 48-54.

[20] D. J. Watts and S. H. Strogatz. "Collective Dynamics of Small-World Networks." Nature 393 (1998), 440-442. 